\title{
Comparison and Analysis of Power Loss Channel and Delay Channel for Dynamic Wireless Network
}

\author{
Navjot Kaur \\ Ambala College of Engineering, \\ Ambala.
}

\author{
Ashok kumar, \\ Ambala College of \\ Engineering, Ambala.
}

\author{
Manju Shrma \\ DAVIET, Jalandhar
}

\begin{abstract}
In this paper we contrast wireless network for power loss channel and delay channel used for data transmission. The topology composed of dynamic wireless composites whose position is set by random function and use Zigbee technology for data transmission with RREQ protocols at varying range of transmission of 300, 400 and 500 units. Paper contrast different wireless channel with different wireless protocols in different conditions and concludes to specific result at particular condition.
\end{abstract}

Keywords - Zigbee, RREQ, Power Loss Channel, Delay Channel, AODV and Visual Sense.

\section{INTRODUCTION}

ZigBee technology is a low data rate, low power consumption, low cost; and remote control applications. And connectivity for equipment that needs battery life as long as several months to several years but does not require data transfer rates as high. The data rate is $250 \mathrm{kbps}$ as $2.4 \mathrm{GHz}$. ZigBee uses a basic master-slave configuration suited to static star networks of many infrequently used devices that small data packets.[1] Other design goals are also maintained in the proposed protocols. As wireless systems are used in more and more settings, and for longer time periods, more accurate characterization of channel parameters is vital to effective and efficient Communication system design.[6] Power Loss Channel (RREQP), Delay Channel (RREQD).Its Route Request message (RREQ) can send in the channel. Each node in a mesh network employing AODV contains a routing table, with entries to the different destination nodes it knows how to reach. A routing table entry is indexed by the destination address, and includes the address of the next hop in the path, the hop count to travel, a destination sequence number, a route neighbor list, and an expiration time. Should a node try to transmit data to another node that is not in its routing table, the node will attempt path discovery. Path discovery is performed by broadcasting a route request (RREQ) packet. The RREQ packet contains several namely source and destination addresses, source and destination sequence numbers, a broadcast ID, and a hop count. The source address and broadcast ID together uniquely identify a RREQ packet. Once the RREQ packet arrives at a node, the node that it has not received this particular RREQ packet; it drops the packet. It then checks its own routing table to see if it knows of a valid route to the destination. A route is valid if the routing table entry's destination sequence number is larger than the one present in the RREQ packet. If the routing table does not contain such a route, the node increments the hop count in the RREQ packet and rebroadcasts it. In order to derive reliable channel models in particular for evaluation channel.
This contribution focused on the analysis of the large scale parameters derived from power and delay. The presented data offers high quality of the acquired data and applicability for high resolution multipath parameter. [3]Wireless traffic may be classified by their delay and loss characteristics. Two common types of traffic are data traffic, which cannot tolerate any loss but has no delay constraint, and real-time traffic, which can tolerate some small amount of loss but has time constraints. It is challenging to handle the second type since both the delay constraint and the loss requirements need to be satisfied. Since the errors in packet transmission cannot be avoided and bursty errors are common. Two common techniques to correct the errors are forward error correction (FEC) and automatic repeat request (ARQ). FEC is often used in either real-time communications or in which feedback channels are not available. A suitable interleaving method, such as block interleaving, which introduces some delay. ARQ has been used to deliver packets in applications that cannot tolerate any error but there is no delay constraint. As the bandwidth of the wireless channel increases, the number of slots available for possible retransmissions within a fixed time period increases. ARQ can perform better than FEC in terms of packet loss probability when the tolerable delay is large, while FEC's performance is better when the delay limit is very small. Traffic sources can be correlated. The delay was estimated by the number of retransmissions, which did not include queuing delay, [4] expired packets were discarded only at the receiver, which introduced inaccuracy.

\section{SYSTEM DESCRIPTION}

\subsection{RREQ Process}

After receiving first RREQ, D broadcasts Nack to stop unnecessary further broadcasting of identical RREQ. After receiving Nack for other nodes, which has not the broad-casted identical RREQ. All IR which have already broad-casted RREQ once, discard Nack of same ID.

$S$ first checks its route table to determine whether it already has a route to $D$. If such a route exists, it can use that route for packet delivery, otherwise route discovery is needed. Route discovery process consists of following processes. In this section we have discussed our two proposed algorithm, with the following set of symbols:

i. $\mathrm{S}=$ Source node, $\mathrm{D}=$ Destination node

ii. $\mathrm{IR}=$ Intermediate Routers or nodes between $\mathrm{S}$ and D.

iii. RREQP = Power Loss Channel (Route Request message), RREQD $=$ Delay Channel (Route Request message). 
iv. RREQ ID.s.id, RREQ ID.dis.id = Route Request id with corresponding extension.

$\mathrm{v} . \mathrm{TR}=$ Transmission Range of a node

vi. Nack $=$ Acknowledgment to ensure that destination has received the RREQ and to inform other non-transmitted nodes not to transmit further identical RREQ and discard it.

\subsection{Packet-Transmission Process}

A wireless communication channel delivering packets from a sender to a receiver is considered. This channel is one of the many channels using the same frequency band supported by a wireless system. Time is divided into fixed-size slots and a packet can be transmitted in each slot. Time-division multiplexing is used and the channel under consideration is regularly allocated a time slot after a fixed period of time. Regular slot allocation is important since the actual delay of a packet can be calculated easily if we know the delay in terms of time slots. The slot-allocation scheme of other channels is not important in this study and it may be the same as that of the channel considered. Packets are generated from the source and are put into a first-in-firstout queue at the sender. The packet at the head of the queue is transmitted to the receiver through the wireless channel, possibly incurring errors along the way. The receiver checks for errors and sends an acknowledgment back to the sender through a feedback channel. The Acknowledgment, which is small in size and highly protected, is assumed to be error free. Since two Successive slots for the same channel are never allocated in time, the acknowledgment is assumed to have been received by the sender before the beginning of its next allocated slot. If the packet has been received correctly, the next packet in the queue is transmitted. If the packet contains errors, retransmission of the same packet takes Place if the delay constraint of the packet is not exceeded. The packet is discarded. The next packet in the queue is transmitted and the cycle repeats. [4] We shall obtain the average delay, packet-loss rate, and power consumption. [5]

\subsection{Power Loss Channel}

This is a model of a wireless channel with a specified power propagation that is evaluated and then multiplied by the power field of the transmit properties before delivery to the receiver. For convenience, a variable named "distance" is available and equal to the distance between the transmitter and the receiver when the power propagation formula is evaluated. It can depend on the distance. The value of the power field should be interpreted as power at the transmitter but power density at the receiver. A receiver may multiply the power density with its efficiency and an area. A receiver can then use the resulting power to compare against a detectable threshold, or to determine signal-to-interference ratio. The transmit properties to transmitter as a power field of type double. The default value provided by this channel is infinity, which when multiplied by any +ve constant which presumably will be above any threshold. Any receiver that is within specified range when transmit is the loss Probability Parameter is set to greater than zero.

\subsection{Delay Channel}

A wireless channel is a specified propagation speed and loss probability. The propagation speed introduces delay that depends on the distance between the transmitter and receiver at the time that the transmitter initiates the communication. This channel model assumes that the duration of the message is effectively zero, so that neither the transmitter Nor the receiver move during the transmission. It also assumes that the receiver does not move during Propagation. It assumes that the propagation of the message is much faster than the node movement.

This is the simple version of protocol so that it simulation as a fast as possible. The modal has the main characteristics of Zigbee, but without separate layer. This version just implements a Power Loss Channel and Delay Channel is very simple AD- Hoc on demand distance vector routing. 


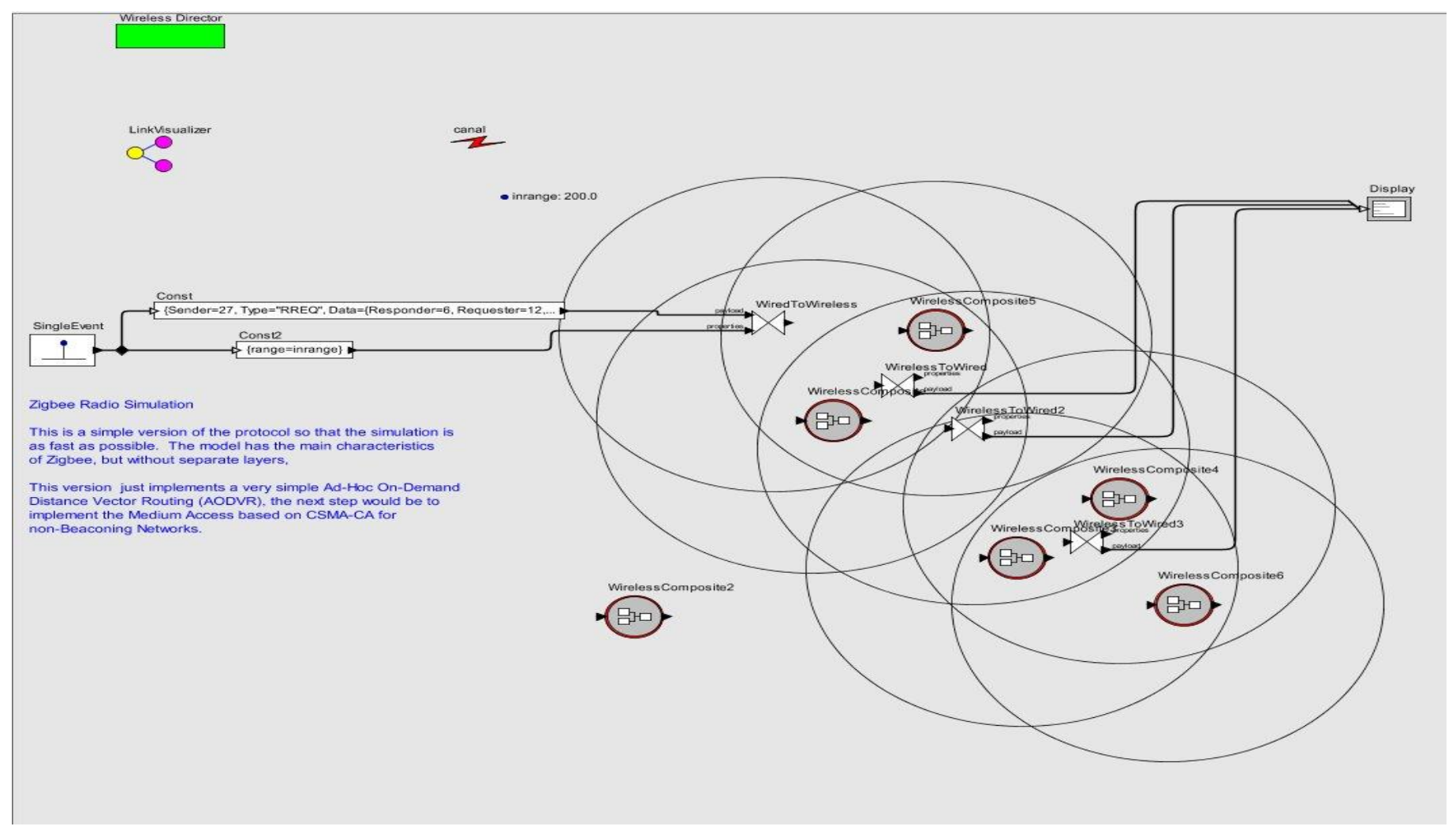

Figure.1Power Loss Channel

Figure.1.1 Properties of Power Loss Channel

\begin{tabular}{|c|c|}
\hline \multicolumn{2}{|l|}{ Edit parameters for PLC } \\
\hline \multirow{6}{*}{$\begin{array}{l}\text { defaultProperties: } \\
\text { name: } \\
\text { lossProbability: } \\
\text { seed: } \\
\text { propagationSpeed: } \\
\text { powerPropagationFactor: }\end{array}$} & \{range $=$ Infinity, power = Infinity $\}$ \\
\hline & \\
\hline & 0.0 \\
\hline & $\mathrm{OL}$ \\
\hline & Infinity \\
\hline & $1.0 /(4 * \mathrm{PI} *$ distance * distance $)$ \\
\hline
\end{tabular}

Commit Add

Remove Restore Defaults 


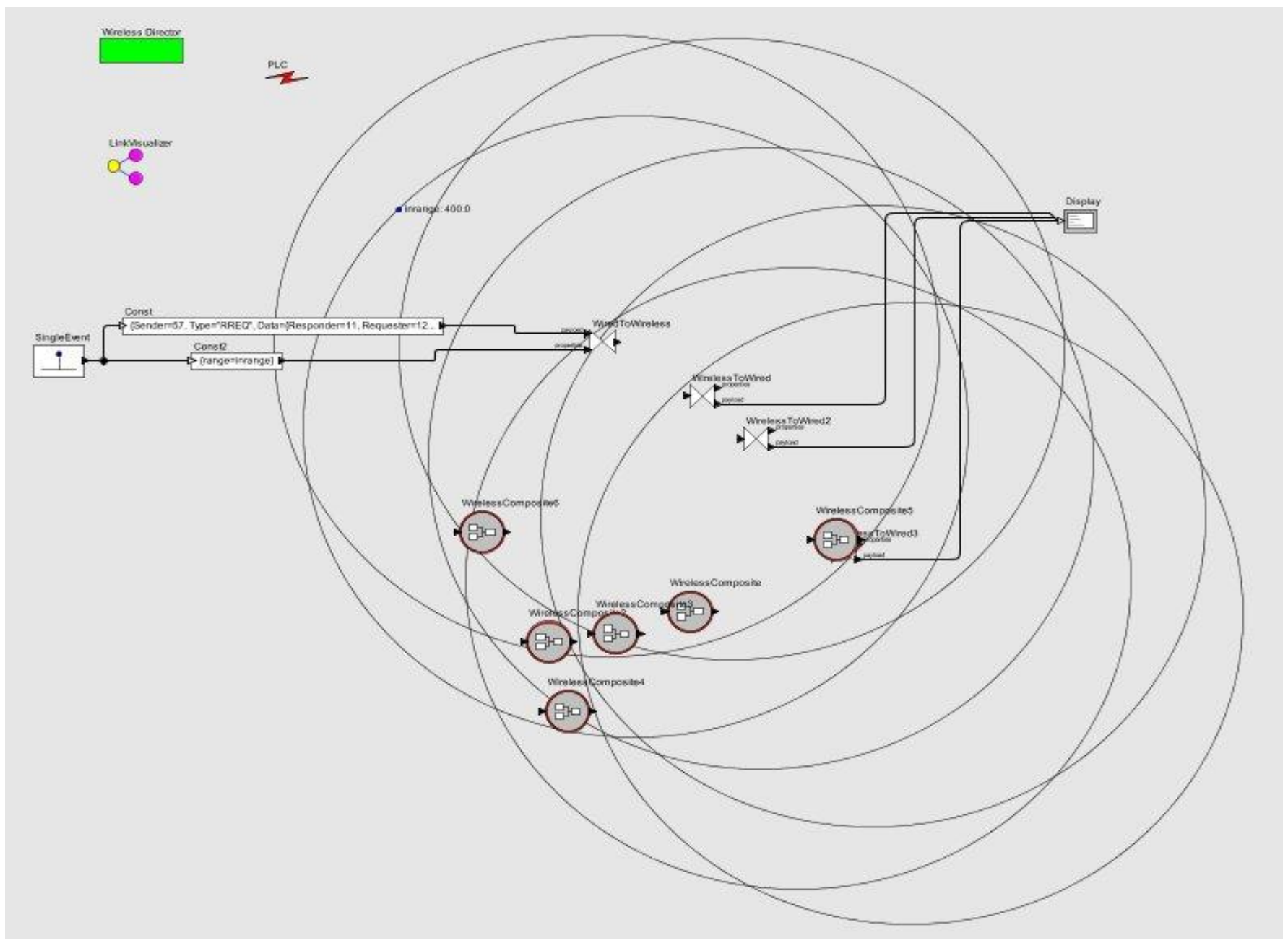

Figure.2 Delay Channel

\begin{tabular}{|c|c|}
\hline Edit parameters for $\mathrm{PLC}$ & \\
\hline defaultProperties: & \} \\
\hline name: & $\mathrm{PLC}$ \\
\hline lossProbability: & 0.5 \\
\hline seed: & $\mathrm{OL}$ \\
\hline propagationSpeed: & Infinity \\
\hline
\end{tabular}

Commit

Add Remove

Restore Defaults Preferences

Help

Cancel

Figure.2.1 Properties of Delay Channel 


\section{RESULTS}

In this section, we present our experimental data in tabular and graphical forms, along with our interpretation of the data. For all the simulations, the same movement models were used, the number of traffic sources was fixed at different ranges $300,400,500$ inrange. The maximum numbers of data packets are to be sending in number of wireless composite of the channels.

The numbers of data packets are send RREQ which has data to be generated for the wireless composite of the channel. It has also generates the packet of wireless composite with the factor of increasing with a growing number of sources. [7] The on-demand of network AODV performed particularly well. The numbers of data packets are send RREQPG and RREQDG which has data to be generated for the wireless composite. It has also generates the packet of wireless composite with the factor of increasing with a growing number of sources. [6] When the number of sources is low, the performance of AODV is similar regardless. Additionally, we discover that the transmission delay would decline with the decrease of packet size. When the size of data packet varies from Retransmission would lead to long transmission delay.

\section{Packets delivery at range of $\mathbf{3 0 0}$}

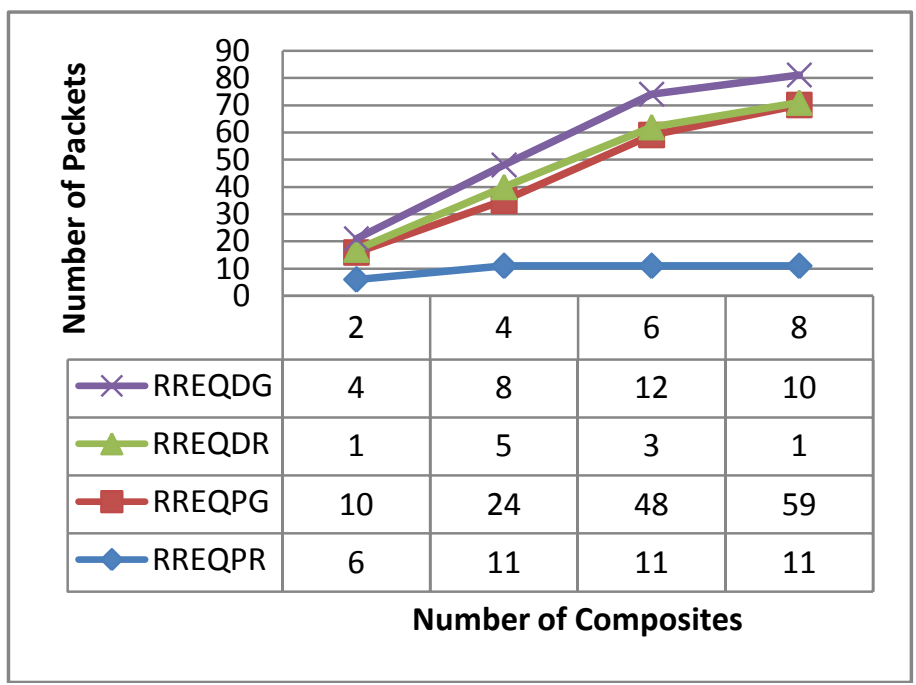

Figure3.1 At Range of 300

2. Packets delivery at range of $\mathbf{4 0 0}$

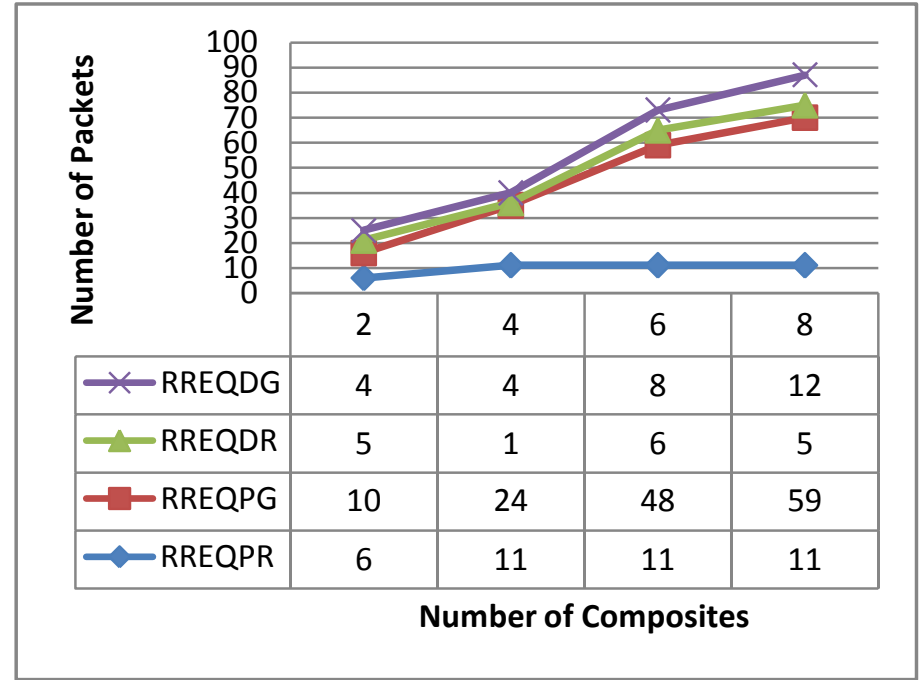

Figure3.2 At Range of 400

\section{Packets delivery at range of 500}

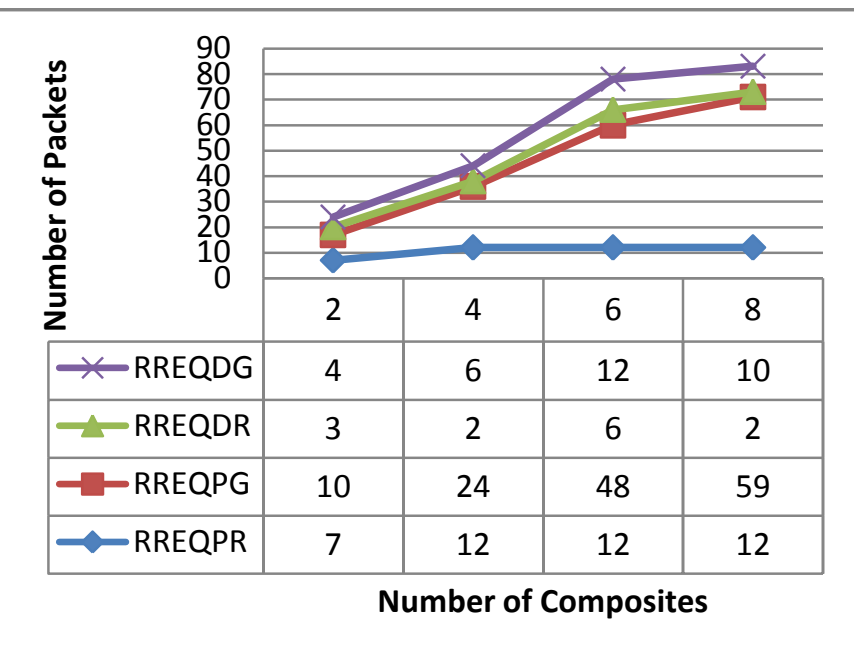

Figure3.3 At Range of 500

Furthermore, immediate retransmissions on a channel that is currently in a deep fade are often useless. The 500 range number of data packets are delivered is better than other ranges. The sometimes advocated idea of postponing retransmissions until the deep fade ends is obviously not a good idea for packets with real-time deadlines. Therefore, it is quite necessary to find some new strategies for lowering transmission delay. [8] The transmission delay will be increased up to 12 time unit; the transmission delay will be very long and approach to infinite. The transmission Delay decreases from 4 time unit. [9]

\section{CONCLUSION}

The results shows that the number of packets generated and received are large in number of delay channel for different ranges of transmission this is due to packet loss probability of 0.5 in power loss channel. The packets generated and received remains less, same and almost constant at all ranges for power loss channel, while shows 
significant difference for delay channel. This comparison can be further extended in future for more number of composites and large range of transmitters.

\section{REFERENCES}

[1] Sinem Coleri Ergen," ZigBee/IEEE 802.15.4 Summary,"Thesis.

[2] Swarnali Hazra University of Calcutta, India,Sanjit Setua University of Calcutta India,"Optimization on Control Overhead in MANET,'International Journal of Computer Applications (0975 - 8887).

[3] Annika Boettcher*, Christian Schneider†, Milan Narandzic $\dagger$, Peter Vary*, and Reiner S. Thomae $\uparrow *$ Institute of Communication Systems and Data Processing, RWTH Aachen University (Germany),"Power and Delay Domain Parameters of Channel Measurements at 2.53 GHzin an Urban Macro Cell Scenario,'IEEE.

[4] Kelvin K. Lee and Samuel T. Chanson," Packet Loss Probability for Bursty Wireless Real-Time Traffic Through Delay Model," IEEE TRANSACTIONS ON VEHICULAR TECHNOLOGY, VOL. 53, NO. 3, MAY 2004.

[5] Department of Electronic Engineering Tsinghua University Beijing, 100084CHINA,"Optimal Delay-Power Tradeoff in Wireless Transmission with Fixed Modulation,'IEEE.

[6] David W. Matolak, Susheel Bokdia Rajendar, Qian Zhang School of Electrical Engineering and Computer Science,"Modeling Wireless Channel Delay Spread Trends," IEEE.

[7] A.F.A. Abidin1, N.S.M. Usop2 and M.K. Yusof 3Faculty of Informatics, Universiti Sultan Zainal Abidin, Kuala Terengganu,
Malaysia,"Performance Comparison of WirelessAd Hoc Routing Protocols," International Journal.

[8] Daniel E. Quevedo, Member, IEEE, Anders Ahlén, Senior Member, IEEE, and Jan Østergaard,"Energy Efficient State Estimation With Wireless Sensors Through the Use of Predictive Power Control and Coding," IEEE.

[9] Weibao Zheng \#, Ming Huang \#2, Jiang Yu\#, Yang Song\#, Jingsong $\mathrm{Hu}$ ','Wireless dual channel model for lower error and delay," IEEE.

[10] Nikola Zogović, Goran Dimić University of Belgrade Institute Mihajlo Pupin Belgrade, Serbia," Channel Loss Based Energy Consumption Model for Low-Power Wireless Communications,'IEEE.

[11] A.Algans, K.I.Pedersen, P.E.Mogensen, "Experimental Analysis of the Joint Statistical Properties of Azimuth Spread, Delay Spread, and Shadow Fading", IEEE Journal on selected Areas in Communication, Vol.20, No.3, 2002.

[12] G. Matz, "On Non-WSSUS Wireless Fading Channels," IEEE Trans Wireless Comm., vol. 4, no. 5, pp. 2465-2478, Sept.2005.

[13] W. Ren, Q. Zhao, and A. Swami "On the Connectivity and Multihop Delay of Ad Hoc Cognitive Radio Networks", IEEE Journal on Selected Areas in Communications (JSAC), vol. 29, no. 4, pp. 805-818, April, 2011.

[14] K. Liu, Q. Zhao, and B. Krishnamachari"Dynamic Multichannel Access with Imperfect Channel State Detection", IEEE Transactions on Signal Processing, vol. 58, No. 5, pp. 2795 2808 , 